\title{
High Dimensional Consistent Digital Segments
}

\author{
Man-Kwun Chiu ${ }^{1}$ and Matias Korman*2 \\ 1 National Institute of Informatics (NII), Tokyo, Japan; and \\ JST, ERATO, Kawarabayashi Large Graph Project, Japan \\ chiumk@nii.ac.jp \\ 2 Tohoku University, Sendai, Japan \\ mati@dais.is.tohoku.ac.jp
}

\begin{abstract}
We consider the problem of digitalizing Euclidean line segments from $\mathbb{R}^{d}$ to $\mathbb{Z}^{d}$. Christ et al. (DCG, 2012) showed how to construct a set of consistent digital segments (CDS) for $d=2$ : a collection of segments connecting any two points in $\mathbb{Z}^{2}$ that satisfies the natural extension of the Euclidean axioms to $\mathbb{Z}^{d}$. In this paper we study the construction of CDSs in higher dimensions.

We show that any total order can be used to create a set of consistent digital rays CDR in $\mathbb{Z}^{d}$ (a set of rays emanating from a fixed point $p$ that satisfies the extension of the Euclidean axioms). We fully characterize for which total orders the construction holds and study their Hausdorff distance, which in particular positively answers the question posed by Christ et al..
\end{abstract}

1998 ACM Subject Classification I.3.5 Computational Geometry and Object Modeling, I.4.1 Digitization and Image Capture

Keywords and phrases Consistent Digital Line Segments, Digital Geometry, Computer Vision

Digital Object Identifier 10.4230/LIPIcs.SoCG.2017.31

\section{Introduction}

Computation in Ancient Greece was rigorously done with ruler and compass using the five axioms of Euclidean geometry. The study of these axioms has had a drastic influence in the development of mathematics. Indeed, the removal of one of them (the fifth one) created non-Euclidean geometries, which have had huge influence on science and technology.

Computers and digital data have nowadays replaced the ruler and compass methods of computation. In order to have a rigorous system of geometric computation in the digital world, it is desirable to establish a set of axioms similar to those of the Euclidean geometry, where we need to replace a line by a Manhattan path in the micro scale that in a macro scale can be seen as a straight line.

There have been several attempts to define digital segments in a two dimensional $n \times n$ grid. The two dimensional bounded space is the most popular case to consider given its many applications in computer vision and computer graphics. Solutions have been proposed from a robust finite-precision geometric computation point of view [6, 8], snap rounding [5], and many more.

A pioneering work by Michael Luby in 1987 [7] introduced an axiomatic approach of the set of digital rays emanating from the origin. He showed that lines should curve by $\Theta(\log n)$ to satisfy a set of axioms analogous to Euclid's axioms (the lower bound proof was given

\footnotetext{
* M. K. was supported in part by the ELC project (MEXT KAKENHI No. 12H00855, 15H02665, and 24106007).
} 
by Håstad). The theory was recently re-discovered by Chun et al. [4] and Christ et al. [3]. Using these results we can define a geometry that satisfies Euclid-like axioms in the two dimensional grid, and only a small bend of the lines will be needed (i.e., $\Theta(\log n)$ in an $n \times n$ grid, a formal definition is given below).

Chun et al. and Christ et al. proposed a $d$-dimensional version of the set of axioms, but unfortunately it is not constructive. That is, they left open how to find a system to generate a complete set of digital segments in $d$-dimensional space that resembles the Euclidean segments. In this paper we provide the first significant step towards answering the question for high dimensions. For the purpose we extend the constructive algorithm of Christ et al. [3] to spaces of arbitrary dimension and study how much of a bend it creates.

\section{Preliminaries}

Let $x_{1}, x_{2}, \ldots, x_{d}$ denote the coordinate axes in $\mathbb{Z}^{d}$, and $p_{i}$ denote the $i$-th coordinate of a point $p \in \mathbb{Z}^{d}$ (for simplicity, from now on all indices are in the set $\{1, \ldots, d\}$ ). Our aim is to construct a digital path for any two points $p, q \in \mathbb{Z}^{d}$ (we denote such a path by $R(p, q)$ ). Ideally, we want $R$ to be constructive and defined in the whole domain, but sometimes we will consider subsets of $\mathbb{Z}^{d} \times \mathbb{Z}^{d}$ instead.

- Definition 1. For any $S \subseteq \mathbb{Z}^{d} \times \mathbb{Z}^{d}$, let $D S(S)$ be a set of digital segments such that $R(p, q) \in D S(S)$ for all $(p, q) \in S$. We say that $D S(S)$ forms a partial set of consistent digital segments on $S$ (partial CDS for short) if for every pair $(p, q) \in S$ it satisfies the following five axioms:

(S1) Grid path property: $R(p, q)$ is a path between $p$ and $q$ under the $2 d$-neighbor topology ${ }^{1}$.

(S2) Symmetry property: $R(p, q)=R(q, p)$.

(S3) Subsegment property: For any $r \in R(p, q)$, we have $R(p, r) \in D S(S)$ and $R(p, r) \subseteq$ $R(p, q)$.

(S4) Prolongation property: There exists $r \in \mathbb{Z}^{d}$, such that $R(p, r) \in D S(S)$ and $R(p, q) \subset$ $R(p, r)$.

(S5) Monotonicity property: For all $i \leq d$ such that $p_{i}=q_{i}$, it holds that every point $r \in R(p, q)$ satisfies $r_{i}=p_{i}=q_{i}$.

These axioms give nice properties of digital line segments analogous to Euclidean line segments. For example, (S1) and (S3) imply that the non-empty intersection of two digital line segments is connected under the $2 d$-neighbor topology. In particular, the intersection between two digital segments is a digital line segment that could degenerate to a single point or even to an empty set. (S5) implies that the intersection with any axis-aligned halfspace is connected, and so on.

Ideally, we want the set $S$ to be as large as possible. A subset of $\mathbb{Z}^{d} \times \mathbb{Z}^{d}$ that is often used for constructions is $S=\{p\} \times \mathbb{Z}^{d}$ (for some $p \in \mathbb{Z}^{d}$ ). We say that a partial CDS on such a set is a consistent digital ray system (CDR for short). Note that this means that we have a method to connect a fixed point $p$ to any other point of $\mathbb{Z}^{d}$. A partial CDS for $S=\mathbb{Z}^{d} \times \mathbb{Z}^{d}$ is called a set of consistent digital segments (CDS for short). Our aim is to create a CDS in $\mathbb{Z}^{d}$, since it is a constructive way to connect any pairs of points.

It is not straightforward how to create a CDS, even when $d=2$. For example, the simple rounding scheme of Euclidean segments to the digital world that is often used in

1 The $2 d$-neighbor topology is the natural one that connects to your predecessor and successor in each dimension. Formally speaking, two points are connected if and only if their $L_{1}$ distance is exactly one. 


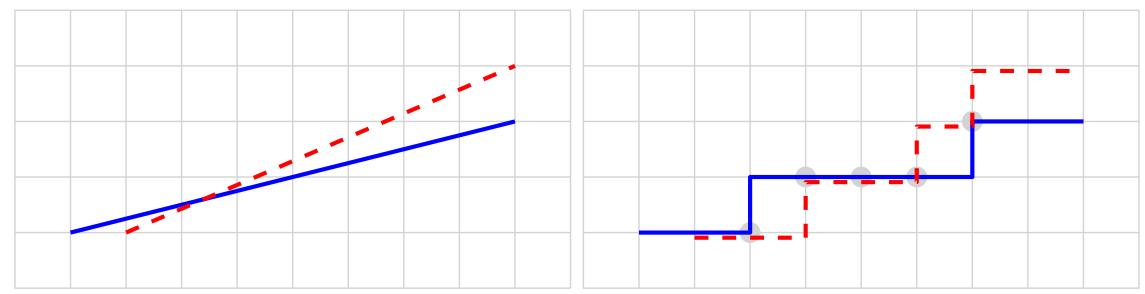

Figure 1 Two different Euclidean line segments and their corresponding digital line segments via a rounding scheme. Note that their intersection in $\mathbb{Z}^{2}$ (highlighted with grey disks) is not connected under the 4-neighbor topology, which implies that the rounding scheme is not consistent.

computer graphics, does not generate a CDS (since axioms are not always preserved, see Figure 1). Another alternative is to use the bounding box approach that makes all moves in one dimension before moving in another one. Although this set of segments is consistent, it will be visually very different from the Euclidean line segments. Thus, the objective is to create a CDS that resembles the Euclidean segments.

The straightness or resemblance between the digital line segment $R(p, q)$ and the Euclidean segment $\overline{p q}$ is often measured using the Hausdorff distance. The Hausdorff distance $H(A, B)$ of two objects $A$ and $B$ is defined by $H(A, B)=\max \{h(A, B), h(B, A)\}$, where $h(A, B)=$ $\max _{a \in A} \min _{b \in B} \delta(a, b)$, and $\delta(a, b)$ is the natural Euclidean distance, given by $\|\cdot\|_{2}$ norm.

- Definition 2. Let $D S(S)$ be a partial CDS. We say that $D S(S)$ has Hausdorff distance $f(n)$ if for all $p, q \in S$ such that $\|p-q\|_{1} \leq n$, it holds that $H(\overline{p q}, R(p, q))=O(f(n))$.

Constructions with smaller Hausdorff distance resemble more the Euclidean segments and thus, are more desirable. Hence, the big open problem in the field is what is the (asymptotically speaking) smallest $f(n)$ function so that we can have a CDS in $\mathbb{Z}^{d}$ ? Or equivalently: what is the asymptotic behavior of the Hausdorff distance of the CDS that best approximates the Euclidean segments? ${ }^{2}$

\subsection{Previous work}

Although the concept of consistent digital segments was first studied by Luby [7], it received renewed interest by the community when it was rediscovered by Chun et al. [4]. The latter showed how to construct a set of consistent digital rays (CDR) in any dimension. The construction satisfies all axioms, including the Hausdorff distance bound:

- Theorem 3 (Theorem 4.4 of [4], rephrased). For any $d \geq 2$ and $p \in \mathbb{Z}^{d}$ we can construct a CDR with $O(\log n)$ Hausdorff distance.

Håstad ${ }^{3}$ and Chun et al. [4] showed that any CDR in two dimensions must have $\Omega(\log n)$ Hausdorff distance. Thus $\log n$ is the smallest possible distance one can hope to achieve. This result was generalized by Christ et al. [3], who shows a correspondence between CDRs in $\mathbb{Z}^{2}$ and total orders on the integers (details on this correspondence is given in Section 3).

\footnotetext{
2 Note that in the original definition of Christ et al. [3], the requirement is for points $p, q \in S$ such that $\|p-q\|_{2} \leq n$. The focus of interest is the two dimensional case, and for any fixed dimension both metrics are equivalent (since $\frac{\sqrt{d}}{d}|| p-q\left\|_{1} \leq\right\| p-q\left\|_{2} \leq\right\| p-q \|_{1}$. However, since we are interested in bounds that depend in the dimension $d$, it is more accurate to measure the distance between $p$ and $q$ with the $L_{1}$ metric.

3 The lower bound was published by Luby, but credit given to Håstad (see Theorem 19 of [7]).
} 
In particular, this correspondence can be used to create a CDS in $\mathbb{Z}^{2}$ that has $O(\log n)$ Hausdorff distance. Note that the $\Omega(\log n)$ lower bound also holds for CDS, so this result is asymptotically tight.

This answers the question of how well can CDSs approximate Euclidean segments in the two dimensional case. However, the question for higher dimensions remains largely open. Although the method of Christ et al. [3] cannot be used to construct CDSs or CDRs in higher dimensions, they show that it can create partial CDSs.

- Theorem 4 (Theorem 16 of [3], rephrased). Let $S=\left\{(x, y): x_{i} \geq y_{i}\right\} \subset \mathbb{Z}^{d} \times \mathbb{Z}^{d}$. We can construct arbitrarily many partial CDSs on $S$.

Note that $S$ contains segments of positive slope (that is, only for the pairs $(p, q)$ such that $q$ is in the first orthant of $p$ ), hence it is roughly a small fraction (roughly $1 / 2^{d-1}$ ) of all possible segments. Other than Theorems 3 and 4, little or nothing is known for three or higher dimensions. Up to date, the only CDS known in three or higher dimensions is the naive bounding box approach (described in Section 3) that has $\Omega(n)$ Hausdorff distance. In particular, it still remains open whether one can create a CDS in $\mathbb{Z}^{d}$ with $o(n)$ Hausdorff distance (for $d>2$ ).

Other research in the topic has focused in the characterization of CDSs in two dimensions. Chowdhury and Gibson [1] gave necessary and sufficient conditions so that the union of CDRs forms a CDS. This characterization heavily uses the correspondence between CDRs and total orders, and thus it was stated in terms of total orders. In a companion paper, the same authors [2] afterwards provided an alternative characterization together with a constructive algorithm. Specifically, they gave an algorithm that, given a collection of segments in an $n \times n$ grid that satisfies the five axioms, computes a CDS that contains those segments. The algorithm runs in polynomial time of $n$. Both results only hold for the two dimensional case.

\section{Other definitions}

Given two points $p, q \in \mathbb{Z}^{d}$ such that $p \neq q$, the slope of $R(p, q)$ is the sign vector $\mathrm{t}=$ $\left(t_{1}, t_{2}, \ldots, t_{d}\right) \in\{+1,-1\}^{d}$, where $t_{i}=+1$ if $p_{i} \leq q_{i}$ and is -1 if $p_{i} \geq q_{i}$. Note that two points have more than one slope when $p_{i}=q_{i}$ for some index $i$. For simplicity, we refer to the slope of $R(p, q)$ (whenever $p$ and $q$ have more than one slope we pick one arbitrarily). Let $T$ be the set containing all $2^{d}$ slopes of $\mathbb{Z}^{d}$. For any two vectors $u, v \in \mathbb{Z}^{d}$, let $u \cdot v$ denote their dot product.

A total order $\theta$ of $\mathbb{Z}$ is a binary relation on all pairs of integers. We denote that $a$ is smaller than $b$ with respect to $\theta$ by $a \prec_{\theta} b$. We say that two elements $a$ and $b$ are consecutive if there is no number between them (i.e., no integer $c$ satisfies $a \prec_{\theta} c \prec_{\theta} b$ ).

We define three operations on total orders: shift, flip and reverse. The shift operation is denoted by $\theta+c$ and is the result of adding a constant value $c$ to each integer without changing their binary relations (that is, $a \prec_{\theta} b$ if and only if $a+c \prec_{\theta+c} b+c$ ). Similarly, the flip of $\theta$ is denoted by $-\theta$ and is the result of changing the sign of all binary relations (that is, $a \prec_{\theta} b$ if and only if $-a \prec_{-\theta}-b$ ). The reverse operation of $\theta$ (denoted by $\theta^{-1}$ ) is the total order resulting in inverting all relationships (that is, $a \prec_{\theta} b$ if and only if $b \prec_{\theta^{-1}} a$ ).

For any $a<b \in \mathbb{Z}$, we will restrict a total order $\theta$ to an interval $[a, b]$ (and denote it by $\theta[a, b])$. For these subsets we also use the same shift, flip and reverse operations whose definitions follow naturally. In particular, observe that $(\theta[a, b])^{-1}=\left(\theta^{-1}\right)[a, b]$, $(\theta[a, b])+c=(\theta+c)[a+c, b+c]$, and $-(\theta[a, b])=(-\theta)[-b,-a]$. Due to lack of space some proofs are deferred to the extended version of the document. 


\subsection{Results overview and paper organization}

We study properties that CDRs and CDSs must satisfy in high dimensions (i.e., $d \geq 3$ ), and show that they behave very differently from the two-dimensional counterparts. In Section 3 we introduce the concept of axis-order. Although not needed in two dimensions, it allows us to extend the total order construction of Christ et al. to higher dimensions.

Given a point $p \in \mathbb{Z}^{d}$, a total order $\theta$ on the integers, and a slope $\mathrm{t}$, we construct a partial CDS which we denote by $\operatorname{TOC}(\theta, p, \mathrm{t})$. This partial CDS contains segments having an endpoint $p$ and slope t. In two dimensions it generates a tree that covers a quadrant whose corner is $p$ (analogously, in higher dimensions it covers an orthant whose apex is $p$ ).

In two dimensions we have $2^{d}=4$ different slopes. Christ et al. [3] showed that we can pick any four total orders, apply each order to a different slope, and the union of the four constructions will be a CDR. In this paper we show that the analogous result does not hold in higher dimensions: fixing the total order for a single orthant uniquely determines the behavior of other orthants.

- Theorem 5 (Necessary and sufficient condition for CDRs). For any $d>2$, point $p \in \mathbb{Z}^{d}$ and set $\left\{\theta_{\mathrm{t}}: \mathrm{t} \in T\right\}$ of $2^{d}$ total orders, $\bigcup_{\mathrm{t} \in T} T O C\left(\theta_{\mathrm{t}}, p, \mathrm{t}\right)$ forms a CDR at $p$ if and only if for any $\mathrm{t}, \mathrm{t}^{\prime} \in T$ it holds that $\theta_{\mathrm{t}}[\mathrm{t} \cdot p, \infty)=\theta_{\mathrm{t}^{\prime}}\left[\mathrm{t}^{\prime} \cdot p, \infty\right)-\mathrm{t}^{\prime} \cdot p+\mathrm{t} \cdot p$, where $T$ is the set containing all possible slopes of $\mathbb{Z}^{d}$.

In particular, there is a unique way of completing the partial $\operatorname{CDS} \operatorname{TOC}(\theta, p, \mathrm{t})$ to a CDR which we denote by $\operatorname{TOC}(\theta, p)$. The next step is to consider the union of several CDRs to obtain a CDS. For the two dimensional case, Christ et al. showed that we can pick $2^{d-1}=2$ total orders, and if we use them consistently for all points of $\mathbb{Z}^{2}$, the result will always be a CDS. Theorem 5 already implies that only one total order can be involved in the construction of CDSs. In Section 4 we observe that not all total orders will create one, and fully characterize which total orders do so.

- Theorem 6 (Necessary and sufficient condition for CDSs). $\theta$ is a total order such that $\bigcup_{p \in \mathbb{Z}^{d}} \operatorname{TOC}(\theta, p)$ forms $a C D S$ if and only if $\theta=\theta+2$ and $\theta=-(\theta+1)^{-1}$.

In particular, this result positively answers the question posed by Christ et al. of whether their approach can be extended to create CDSs in higher dimensions (posed in a preliminary version of [3]).

The main difference between two dimensional and higher dimensional spaces is that the construction for two different slopes has a larger portion in common. In two dimensions, two quadrants share at most a line (whose behavior is unique because of the monotonicity axiom), but in general orthants may share a subspace of dimension $d-1$. The total orders associated to each orthant must behave similarly within the subspace, which creates some dependency between the total orders. More importantly, each orthant shares subspaces with other orthants, and so on. This cascades creating common dependencies that cycle back to the original orthant and highly constrain the total orders. In Section 6 we discuss this dependency and argue that variations of this construction will also have the same necessary and sufficient conditions.

\section{Extending the total order construction to higher dimensions}

In this section we use a total order to construct a CDR in $\mathbb{Z}^{d}$. We start by reviewing the construction of Christ et al. [3] for $\mathbb{Z}^{2}$. Given a total order $\theta$ and two points $p=\left(p_{1}, p_{2}\right), q=$ $\left(q_{1}, q_{2}\right) \in \mathbb{Z}^{2}$ such that $q_{1} \geq p_{1}$ and $q_{2} \geq p_{2}$, we view the digital segment $R(p, q)$ as a collection 


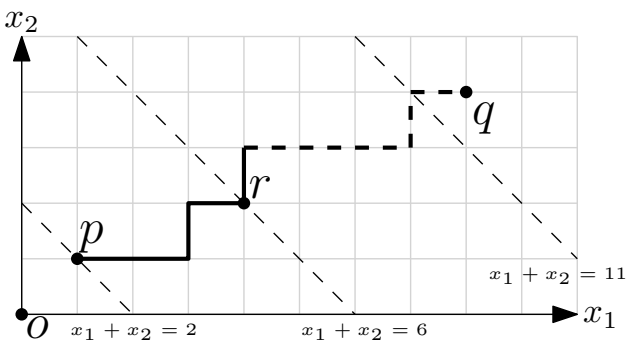

Figure 2 Example of the construction of Christ et al. in $\mathbb{Z}^{2}$. Given $p=(1,1), q=(8,4)$ and a total order $\theta$ such that $\theta[2,11]=5 \prec 3 \prec 2 \prec 7 \prec 9 \prec 8 \prec 11 \prec 10 \prec 6 \prec 4$. The path must perform $q_{1}-p_{1}=7$ steps in the $x_{1}$ direction and $q_{2}-p_{2}=3$ steps in the $x_{2}$ direction. Since $p_{1}+p_{2}=2$ and 2 is among the 7 smallest elements in $\theta[2,11]$, it moves in the $x_{1}$ direction. Similarly, at point $r=(4,2)$, the path will move in $x_{2}$ direction because $r_{1}+r_{2}=6$ is among the 3 largest elements of $\theta[2,11]$. Observe that, for any $c \in[2,11]$ there is a unique point $m$ in the path such that $m_{1}+m_{2}=c$.

of steps that form a path from $p$ to $q$. Due to the monotonicity property, in each step the path increases either the first or second coordinate by one. Clearly, this path must do $q_{1}+q_{2}-p_{1}-p_{2}$ steps, out of which $q_{1}-p_{1}$ are in the $x_{1}$ coordinate (and the remaining ones in the $x_{2}$ coordinate). The choice of which steps we move in which coordinate depends on $\theta$ : assume that after moving several steps we have reached some intermediate point $\left(r_{1}, r_{2}\right)$. Then, we check whether or not the number $r_{1}+r_{2}$ is among the $q_{1}-p_{1}$ smallest elements of $\theta\left[p_{1}+p_{2}, q_{1}+q_{2}-1\right]$. If so, we move from $\left(r_{1}, r_{2}\right)$ in the $x_{1}$ coordinate. Otherwise we do so in the $x_{2}$ coordinate (see an example in Figure 2).

All of the segments created this way have slope $(+1,+1)$. In a similar way, we can pick a total order to define the segments emanating from $p$ with slope $(+1,-1),(-1,+1)$ and $(-1,-1)$. We emphasize that there is no dependency between the total orders: the choice of total order for one slope has no impact on the available options for the others. Moreover, any four choices will result in a CDR (similarly, any CDR in $\mathbb{Z}^{d}$ is associated with $2^{d}$ total orders of $\mathbb{Z}$, one for each slope). As mentioned before, this independence between quadrants does not hold in higher dimensions.

\subsection{Constructing a CDR in $\mathbb{Z}^{d}$ from a total order}

The construction of Christ et al. explains how to construct segments of slope $(+1,+1)$ in $\mathbb{Z}^{2}$ (or equivalently, for points in the first quadrant). The segments of different slopes are obtained via symmetry. In higher dimensions it will be useful to have an explicit way to construct segments of any slope. Thus, we first generalize the method of Christ et al. for any orthant.

In order to get an idea of our approach, we first look at the folklore bounding box approach to construct a CDS. When defining the path between point $p$ and point $q$, we consider the minimum bounding box formed by the two points. The point with smaller $x_{1}$ coordinate will move in the $x_{1}$ coordinate until reaching the $x_{1}$ coordinate of another point. Afterwards, the one with smaller $x_{2}$ coordinate will move in the $x_{2}$ coordinate, and so on until the two points meet (see Figure 3).

So, if $d=3$, for any segment whose slope is $(+1,+1,+1)$ we first do all the movements in the $x_{1}$ coordinate, then $x_{2}$ coordinate, and finally in the $x_{3}$ coordinate. However, if the segment has slope $(+1,-1,-1)$, then the bounding box CDS will travel first in the $x_{1}$ coordinate, then $x_{3}$ and finally $x_{2}$. Intuitively speaking, even though in both cases we are 


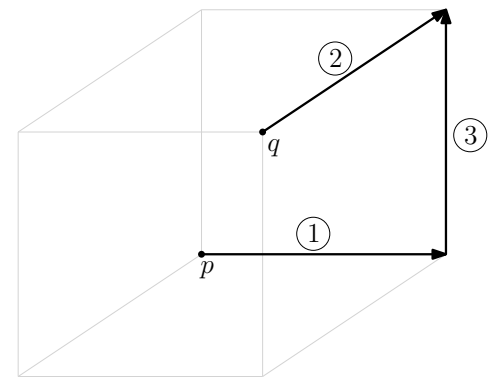

Figure 3 Example of the bounding box approach in $\mathbb{Z}^{3} \cdot p=(0,3,0)$ and $q=(3,0,3)$. The number in each circle indicates the order in which we execute the movements.

performing the same steps (i.e, we use the natural order $0 \prec 1 \prec 2 \prec 3 \prec \ldots$ ), the order in which we execute each dimension is slightly different (or equivalently, the total order is being interpreted differently). We model this difference in interpretation through a new concept which we call axis-order.

Given a slope $\left(t_{1}, t_{2}, \ldots, t_{d}\right)$, let $a_{1}, \ldots a_{k}$ be the indices of the coordinates with positive value in increasing order (that is, $t_{i}=+1$ if and only if $i=a_{j}$ for some $j \leq k$ ). Similarly, let $b_{1}, \ldots b_{d-k}$ be the indices of the coordinates with negative value in decreasing order. Then, the axis-order of $\left(t_{1}, t_{2}, \ldots, t_{d}\right)$ is $x_{a_{1}}, x_{a_{2}}, \ldots, x_{a_{k}}, x_{b_{1}}, \ldots, x_{b_{d-k}}$. For example, the axis-order of $(-1,+1,+1)$ is $x_{2}, x_{3}, x_{1}$, and the axis-order of $(+1,-1,+1)$ is $x_{1}, x_{3}, x_{2}$. As we will see later, it will be useful to consider subspaces of $\mathbb{Z}^{d}$. We observe a property that follows from the definition of axis-order.

- Observation 7. Let $a_{1}, \ldots a_{k}$ be a sequence of indices such that $a_{1}<\ldots<a_{k}$, and let $\mathrm{t}, \mathrm{t}^{\prime} \in\{-1,1\}^{d}$ be two slopes such that $\mathrm{t}_{a_{i}}=\mathrm{t}_{a_{i}}^{\prime}$ (for all $i \leq k$ ). Then, $\mathrm{t}$ and $\mathrm{t}^{\prime}$ have the same axis-order $\tau$ restricted to a subspace $\mathcal{H}$ spanned by $\left\{x_{a_{1}}, x_{a_{2}}, \ldots, x_{a_{k}}\right\}$. Moreover, the axis-order of $-\mathrm{t}$ and $-\mathrm{t}^{\prime}$ restricted to $\mathcal{H}$ is the reverse of $\tau$.

With the help of axis-order we can extend the two dimensional construction to higher dimensions. Given a point $p=\left(p_{1}, \ldots, p_{d}\right) \in \mathbb{Z}^{d}$, a total order $\theta$ and a slope $\mathrm{t}$, we construct the set of rays emanating from $p$ with that slope. Define the orthant $\mathcal{O}_{\mathrm{t}}(p)=\left\{q \in \mathbb{Z}^{d}: \mathrm{t}_{i} \cdot q_{i} \geq\right.$ $\left.\mathrm{t}_{i} \cdot p_{i}\right\}$ : by definition, the segment from $p$ to any point in $\mathcal{O}_{\mathrm{t}}(p)$ has slope $t$. Also, let $x_{a_{1}}, x_{a_{2}}, \ldots$ be the axis-order of $t$.

For any point $q=\left(q_{1}, \ldots, q_{d}\right) \in \mathcal{O}_{\mathrm{t}}(p)$ we construct the segment $R(p, q)$. Similar to the two dimensional case, the path from $p$ to $q$ must do $t \cdot q-\mathrm{t} \cdot p$ steps, out of which $\left|p_{1}-q_{1}\right|$ will be in the first coordinate, $\left|p_{2}-q_{2}\right|$ in the second, and so on. We traverse through intermediate points, each time increasing the inner product with $t$ by one. At each intermediate point $r$, we check the position of $\mathrm{t} \cdot r$ in $\theta[\mathrm{t} \cdot p, \mathrm{t} \cdot q-1]$; if it is among the $\left|p_{a_{1}}-q_{a_{1}}\right|$ smallest elements in $\theta[\mathrm{t} \cdot p, \mathrm{t} \cdot q-1]$ then we move in the $x_{a_{1}}$ coordinate. Otherwise, if it is among the smallest $\left|p_{a_{1}}-q_{a_{1}}\right|+\left|p_{a_{2}}-q_{a_{2}}\right|$ elements we move in $x_{a_{2}}$, and so on.

For example, if the total order $\theta$ satisfies $3 \prec_{\theta} 1 \prec_{\theta} 5 \prec_{\theta} 7 \prec_{\theta} 9 \prec_{\theta} 8 \prec_{\theta} 6 \prec_{\theta} 4 \prec_{\theta} 2 \prec_{\theta} 0$, $p=(0,0,0)$ and $q=(2,-3,5)$, the slope is $(+1,-1,+1)$, axis-order is $x_{1}, x_{3}, x_{2}$. So we must look at $\theta[p \cdot(+1,-1,+1), q \cdot(+1,-1,+1)-1]=\theta[0,9]$. In this total order the number $(+1,-1,+1) \cdot(0,0,0)=0$ is the largest element in $\theta[0,9]$, so we move from $(0,0,0)$ in the $x_{2}$ coordinate to point $(0,-1,0)$. At point $(0,-1,0)$ the number $(+1,-1,+1) \cdot(0,-1,0)=1$ is the second smallest element in $\theta[0,9]$, so we move in the $x_{1}$ coordinate, and so on. Overall the path is $(0,0,0) \rightarrow(0,-1,0) \rightarrow(1,-1,0) \rightarrow(1,-2,0) \rightarrow(2,-2,0) \rightarrow(2,-3,0) \rightarrow$ $(2,-3,1) \rightarrow(2,-3,2) \rightarrow(2,-3,3) \rightarrow(2,-3,4) \rightarrow(2,-3,5)$. 
- Definition 8. For any point $p \in \mathbb{Z}^{d}$, slope $\mathrm{t}$, and total order $\theta$, we call the collection of segments $\left\{R(p, q): q \in \mathcal{O}_{\mathrm{t}}(p)\right\}$ the total order construction of $\theta$ (centered at $p$ ) for the slope $\mathrm{t}$, and denote it by $\operatorname{TOC}(\theta, p, \mathrm{t})$.

\subsection{Properties of the total order construction}

- Lemma 9 (Translation Lemma). For any $p \in \mathbb{Z}^{d}$, slope $\mathrm{t}$ and total order $\theta$, the set of segments in $\operatorname{TOC}(\theta, p, \mathrm{t})$ is the translated copy of the set of segments in $\operatorname{TOC}(\theta-\mathrm{t} \cdot p, o, \mathrm{t})$, where $o$ is the origin.

- Lemma 10. For any $p \in \mathbb{Z}^{d}$, slope $\mathrm{t}$ and total order $\theta$, the set of segments in TOC $(\theta, p, \mathrm{t})$ forms a partial $C D S$ on $\{p\} \times \mathcal{O}_{\mathbf{t}}(p)$.

Proof. This statement is a particular case of Theorem 4: we are interested in segments of a single slope emanating from a fixed point, whereas Theorem 4 only requires segments of a fixed slope. The proof given by Christ et al. [3] is for slope $(+1, \ldots,+1)$, but the arguments extend naturally for the general case.

Let $\theta_{0}$ be the natural order on the integers (that is, $\theta_{0}=\{\ldots \prec-1 \prec 0 \prec 1 \prec 2 \prec \ldots\}$ ). Fix any point $p \in \mathbb{Z}^{d}$ and apply the total order construction $T O C(\theta, p, \mathrm{t})$ to all slopes. Similarly, let $\theta_{1}$ be result of swapping the position of -1 and -2 in $\theta_{0}$ (i.e., $\theta_{1}=\{\ldots \prec$ $-1 \prec-2 \prec 0 \prec 1 \prec 2 \ldots\})$. Let $\mathcal{C}_{0}(p)$ and $\mathcal{C}_{1}(p)$ the union of segments created with each total order, respectively.

- Proposition 11. $\mathcal{C}_{0}(p)$ is a $C D R$ that is included in the bounding box $C D S$ whereas $\mathcal{C}_{1}(p)$ is not a $C D R$.

\subsection{Gluing orthants to obtain CDRs}

The second example of Proposition 11 shows an example of a total order that cannot be applied everywhere to form a CDR. Theorem 5 stated in Section 2.2 shows the relationship that total orders in different slopes must satisfy in order to create a CDR. Intuitively speaking, this correlation is so strong that choosing one total order effectively fixes the rest. The remainder of this section is dedicated to proving this interdependency. We start by showing the proof of one implication of the equivalence.

- Lemma 12 (Necessary condition for CDRs). Let $p \in \mathbb{Z}^{d}$ and $\left\{\theta_{\mathrm{t}}: t \in T\right\}$ be a set of $2^{d}$ total orders such that $\bigcup_{\mathrm{t} \in T} T O C\left(\theta_{\mathrm{t}}, p, \mathrm{t}\right)$ forms a CDR. Then, for any $\mathrm{t}, \mathrm{t}^{\prime} \in T$, it holds that $\theta_{\mathrm{t}}[\mathrm{t} \cdot p, \infty)=\theta_{\mathrm{t}^{\prime}}\left[\mathrm{t}^{\prime} \cdot p, \infty\right)-\mathrm{t}^{\prime} \cdot p+\mathrm{t} \cdot p$.

Proof (Sketch). We prove the statement by contradiction. That is, assume that there exist two slopes $\mathrm{t}, \mathrm{t}^{\prime}$ such that $v \prec_{\theta_{\mathrm{t}}} v^{\prime}$ but $v^{\prime}-\mathrm{t} \cdot p+\mathrm{t}^{\prime} \cdot p \prec_{\theta_{\mathrm{t}^{\prime}}} v-\mathrm{t} \cdot p+\mathrm{t}^{\prime} \cdot p$. Without loss of generality, we can choose $t$ and $t^{\prime}$ so that the corresponding orthants share a two-dimensional plane (pick a sequence of intermediate orthants so that pairwise they do, and look at the first time in which the equality is not satisfied). We pick a point $q$ such that $R(p, q)$ has both slope $\mathrm{t}$ and $\mathrm{t}^{\prime}$, and look at $R(p, q)$ from both the viewpoints of $\operatorname{TOC}\left(\theta_{\mathrm{t}}, p, \mathrm{t}\right)$ and $\operatorname{TOC}\left(\theta_{\mathrm{t}^{\prime}}, p, \mathrm{t}^{\prime}\right)$.

Along the path $R(p, q)$ we look at two intermediate points $r$ and $r^{\prime}$. The main feature of these points is that the behavior of $R(p, q)$ at those points depends on the positions of $v$ and $v^{\prime}$ in $\theta_{\mathrm{t}}$ (if we look at it from the viewpoint of $\operatorname{TOC}\left(\theta_{\mathrm{t}}, p, \mathrm{t}\right)$ ). Since $v \prec_{\theta_{\mathrm{t}}} v^{\prime}$, we can choose $q$ in a way that the path will move in different directions at the two points. Then, we study the same segment from the viewpoint of the other orthant. In this case, the behavior of the same intermediate points will depend on the positions of $v^{\prime}-\mathrm{t} \cdot p+\mathrm{t}^{\prime} \cdot p$ and $v-\mathrm{t} \cdot p+\mathrm{t}^{\prime} \cdot p$ in the shifted total order instead. Thus, if the relationships are reversed, the two paths behave differently and in particular we cannot have a CDR. 
- Lemma 13 (Sufficient condition for CDRs). For any point $p \in \mathbb{Z}^{d}$, let $\left\{\theta_{\mathrm{t}}: t \in T\right\}$ be a set of $2^{d}$ total orders such that $\theta_{\mathrm{t}}[\mathrm{t} \cdot p, \infty)=\theta_{\mathrm{t}^{\prime}}\left[\mathrm{t}^{\prime} \cdot p, \infty\right)-\mathrm{t}^{\prime} \cdot p+\mathrm{t} \cdot p$ for any $\mathrm{t}, \mathrm{t}^{\prime} \in T$. Then, $\bigcup_{\mathrm{t} \in T} \operatorname{TOC}\left(\theta_{\mathrm{t}}, p, \mathrm{t}\right)$ forms a $C D R$.

This completely characterizes the CDRs that can be made with the total order construction in $\mathbb{Z}^{d}$. For any point $p$, slope $\mathrm{t}$ and total order $\theta$, there is a unique CDR that can be created in this way and contains $\operatorname{TOC}(\theta, p, \mathrm{t})$. Since the choice of slope is not important, let $\operatorname{TOC}(\theta, p)$ be the unique CDR that contains $\operatorname{TOC}(\theta, p,(+1, \ldots,+1))$.

- Corollary 14. For any $p \in \mathbb{Z}^{d}$ there exist arbitrarily many CDRs with $O(\log n)$ Hausdorff distance.

Proof. An explicit construction of a single $\mathrm{CDR}$ in $\mathbb{Z}^{d}$ with $O(\log n)$ Hausdorff distance was given by Chun et al. [4]. They showed that the CDR generated using the Van der Corput sequence [9] as total order has low Hausdorff distance (for any dimension). Christ et al. [3] extended the result showing that the straightness is asymptotically same as the discrepancy of the permutation corresponding to the total order, which is known to be $\Theta(\log n)$. Moreover, for any total order $\theta$ with low discrepancy, it holds that $\theta+k$ has low discrepancy (for any $k \in \mathbb{Z}$ ), so the arguments for $d=2$ extend directly to the higher dimension construction. Thus, we omit them.

\section{Necessary and sufficient conditions for CDSs}

Next we focus our attention to constructing CDSs. Christ et al. [3] showed that if we apply the same total order construction to all points of $\mathbb{Z}^{2}$ we get a collection of CDRs whose union is always a CDS. For any total order $\theta$, let $T O C(\theta)=\bigcup_{p \in \mathbb{Z}^{d}} T O C(\theta, p)$. Unlike the two dimensional case, the construction $\operatorname{TOC}(\theta)$ does not always yield a CDS in higher dimensions. Theorem 6 stated in Section 2.2 gives necessary and sufficient conditions that the total order must satisfy.

Recall that in principle, we allow different orthants (except $(+1, \ldots,+1)$ ) to have different total orders in this construction. For any point $p \in \mathbb{Z}^{d}$ and slope $\mathrm{t}$, let $\theta_{\mathrm{t}}^{p}$ be the total order associated to point $p$ and slope $\mathrm{t}$ in $\operatorname{TOC}(\theta)$. Since $\operatorname{TOC}(\theta)$ in particular contains $\operatorname{TOC}(\theta, p)$, Theorem 5 gives a relationship between $\theta$ and $\theta_{\mathrm{t}}^{p}$. We give a stronger bound on that relationship as well.

Theorem 15. If $\theta$ is a total order such that TOC $(\theta)$ forms a $C D S$, then for any $p \in \mathbb{Z}^{d}$ and slope $\mathrm{t}$ it holds that $\theta_{\mathrm{t}}^{p}[\mathrm{t} \cdot p, \infty)=\theta[\mathrm{t} \cdot p, \infty)$. In particular, $\operatorname{TOC}\left(\theta_{\mathrm{t}}^{p}, p, \mathrm{t}\right)=\operatorname{TOC}(\theta, p, \mathrm{t})$.

This shows that, if we want to create a CDS in this fashion, we must use the same total order $\theta$ for all points and all slopes. Again, this contrasts with the $d=2$ case where we can combine any two total orders for slopes $(+1,+1)$ and $(+1,-1)$. Christ et al. $[3]$ showed that if we repeat the construction for all points of $\mathbb{Z}^{2}$ the union will form a CDS. The remainder of this section is dedicated to showing Theorems 6 and 15 .

\subsection{Two dimensional preliminaries}

We will often consider two dimensional subspaces and find some requirements that extend to the whole space. Thus, we first show a subtree property that CDS in $\mathbb{Z}^{2}$ must satisfy. Consider any point $p \in \mathbb{Z}^{2}$, slope $\mathrm{t}$, point $q \in T O C\left(\theta_{\mathrm{t}}^{p}, p, \mathrm{t}\right)$ such that $q \neq p$, and all points $r \in \mathbb{Z}^{2}$ such that $R(p, r)$ passes through $q$. This set of points (and their paths to $q$ ) form a 


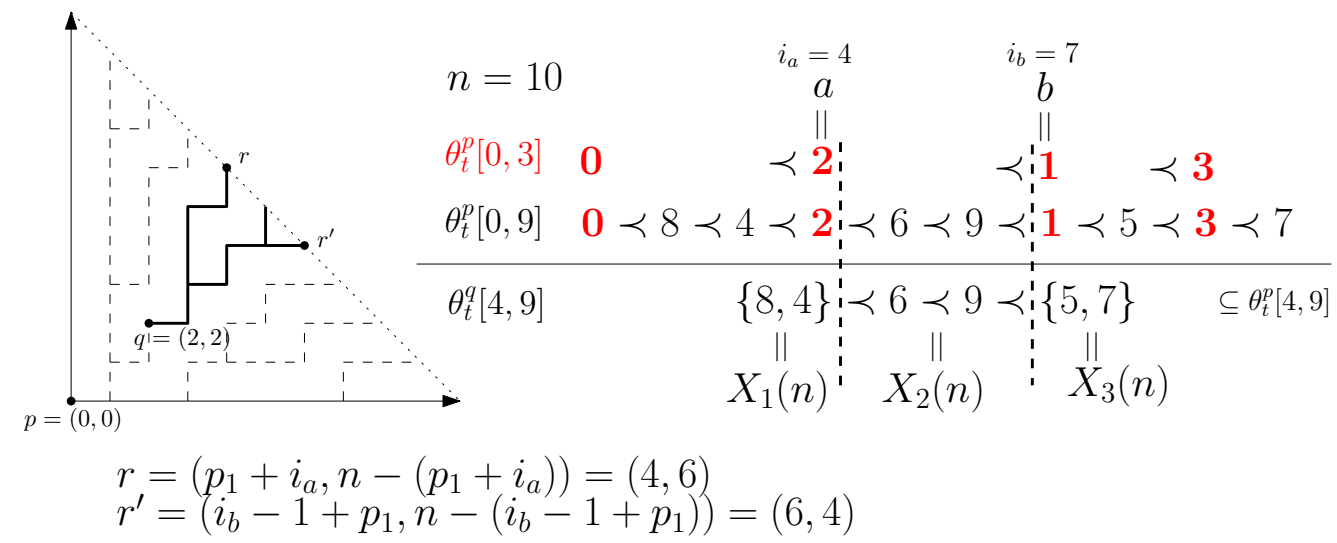

Figure 4 Example of the subtree property. (left) geometric interpretation of the subtree property. The paths to $p$ that pass through $q$ impose a constraint on $\theta_{\mathrm{t}}^{q}$. In particular, a point in the diagonal $x_{1}+x_{2}=n$ will pass through $q$ if and only if it is between $r$ and $r^{\prime}$ (highlighted points in the figure). (right) implications in the total order of $\theta_{\mathrm{t}}^{q}$. In red bold we highlight the points that belong to the left interval. The points in the right interval are classified into the three sets $X_{1}(n), X_{2}(n)$ and $X_{3}(n)$ according to their positions (left of $a$, right of $b$, or in between). The fact that the subtree of $q$ (black in the left figure) has to be preserved in $q$ implies many relationships for $\theta_{\mathrm{t}}^{q}$ that are shown in the third line.

subtree of $\operatorname{TOC}\left(\theta_{\mathrm{t}}^{p}, p, \mathrm{t}\right)$. The same tree must be part of $\operatorname{TOC}\left(\theta_{\mathrm{t}}^{q}, q, \mathrm{t}\right)$ or it would violate (S3) (see Figure 4, left).

We express this subtree property in terms of total orders $\theta_{\mathrm{t}}^{p}$ and $\theta_{\mathrm{t}}^{q}$. Assume $\mathrm{t}=(+1,+1)$, let $s_{1}, s_{2} \geq 0$ be integers such that $q=p+\left(s_{1}, s_{2}\right)$, and let $n$ be any number such that $n>s_{1}+s_{2}$. We will consider the restriction of the total order $\theta_{\mathrm{t}}^{p}$ to three intervals: $[\mathrm{t} \cdot p, \mathrm{t} \cdot q-1]$, $[\mathrm{t} \cdot q, \mathrm{t} \cdot p+n-1]$, and $[\mathrm{t} \cdot p, \mathrm{t} \cdot p+n-1]$. Note that the union of the first two forms the third one. In order to reduce notation we call them the left, the right, and the complete intervals. Similarly, we call $\theta_{\mathrm{t}}^{p}[\mathrm{t} \cdot p, \mathrm{t} \cdot q-1], \theta_{\mathrm{t}}^{p}[\mathrm{t} \cdot q, \mathrm{t} \cdot p+n-1]$, and $\theta_{\mathrm{t}}^{p}[\mathrm{t} \cdot p, \mathrm{t} \cdot p+n-1]$ the left order, the right order and the complete order. The subtree property says that many inequalities in the right order must also hold in $\theta_{\mathrm{t}}^{q}$.

First assume that $s_{1}, s_{2} \neq 0$; let $a$ and $b$ be the $s_{1}$-th and $\left(s_{1}+1\right)$-th smallest numbers in the left order, respectively. By definition, these two numbers are consecutive in the left order, but they need not be in the complete order (i.e., there could be numbers from the right interval).

Let $i_{a}$ and $i_{b}$ be the positions of $a$ and $b$ in the complete order, respectively. We partition the numbers of the right interval into three groups, depending on whether they are $(i)$ smaller than $a,(i i)$ larger than $a$ and smaller than $b$, or (iii) larger than $b$ (all these comparisons are with respect to $\theta_{\mathrm{t}}^{p}$ ). Let $X_{1}(n), X_{2}(n)$, and $X_{3}(n)$ be the three sets, respectively (see Figure 4).

Before giving the subtree property we extend the definitions of these three sets for the cases in which $s_{1}$ and $s_{2}$ can be zero. If $s_{1}=0$ then $a$ and $i_{a}$ are not well defined (similarly, $b$ and $i_{b}$ are not defined when $\left.s_{2}=0\right)$. In the first case we set $i_{a}=0, X_{1}(n)=\emptyset$ and classify the numbers of the right interval into $X_{2}(n)$ and $X_{3}(n)$ depending on whether they are smaller or larger than $b$. Similarly, if $i_{b}$ is not defined, we set $i_{b}=n+1, X_{3}(n)=\emptyset$, and numbers are be split into the two sets $X_{1}(n)$ and $X_{2}(n)$.

The following lemma characterizes the points whose path to/from $p$ passes through $q$ in the quadrant of $(+1,+1)$. 
- Lemma 16. For any $n>s_{1}+s_{2}$, let $r \in \mathbb{Z}^{2}$ be a point such that $r_{1}+r_{2}=p_{1}+p_{2}+n$. The path $R(p, r)$ passes through $q$ if and only if $r_{1} \geq q_{1}, r_{2} \geq q_{2}$ and $i_{a} \leq r_{1}-p_{1} \leq i_{b}-1$.

- Lemma 17 (The subtree property). For any $n>s_{1}+s_{2}$ and $u, v \in[\mathrm{t} \cdot q, \mathrm{t} \cdot p+n-1]$, the following relationships must hold in $\theta_{\mathrm{t}}^{q}$ :

- $u \prec_{\theta_{\mathrm{t}}^{q}} v$ for all $u \in X_{1}(n)$ and $v \in X_{2}(n)$,

- $u \prec_{\theta_{\mathrm{t}}^{q}} v$ for all $u \in X_{1}(n) \cup X_{2}(n)$ and $v \in X_{3}(n)$,

- $u \prec_{\theta_{\mathrm{t}}^{q}} v$ for all $u, v \in X_{2}(n)$ such that $u \prec_{\theta_{\mathrm{t}}^{p}} v$.

- Remark. Although we have stated the subtree property for slope $(+1,+1)$, it is straightforward to see that this result extends to other ones. We stick to this notation for simplicity of exposition, although we will afterwards use it for negative slope as well.

\subsection{Application in high dimensional spaces}

With the subtree property we can show the first necessary condition of Theorem 6 .

Lemma 18. Let $\theta$ be a total order such that TOC $(\theta)$ forms a CDS. Then, $\theta=\theta+2$.

Proof. We first give a birdseye overview of the proof: choose an arbitrary $\lambda \in \mathbb{Z}$ and consider the affine plane $\mathcal{H}=\left\{x_{3}=\lambda, x_{4}=0, \ldots, x_{d}=0\right\}$. In this plane we look at the origin $p=(0,0)$, and points $q=(0,-1)$ and $r=(-1,0)$ (see Figure 5, left). In particular, we look at the third quadrant (the one with slope $(-1,-1))$ : first, from Theorem 5 we know that $\theta_{(-1,-1)}^{p}$ must coincide with $\theta$ (on the interval $[\lambda, \infty)$ ).

We apply the subtree property from $p$ to $q$ and $r$; the key property is that both $\theta_{(-1,-1)}^{q}$ and $\theta_{(-1,-1)}^{r}$ coincide with $\theta+2$ on the interval $[\lambda+1, \infty)$. Moreover, all paths to $p$ must pass through either $q$ or $r$, which in particular implies that all inequalities from $\theta_{(-1,-1)}^{p}$ must also be preserved in either $\theta_{(-1,-1)}^{q}$ or $\theta_{(-1,-1)}^{r}$. By combining all of these properties, we show that $\theta$ coincides with $\theta+2$ on the interval $[\lambda+1, \infty)$. The result works for any value of $\lambda$, so when $\lambda \rightarrow-\infty$ we get $\theta=\theta+2$ as claimed.

More formally, pick any $\lambda \in \mathbb{Z}$ and consider the points $p=(0,0, \lambda, 0, \ldots 0), q=$ $(0,-1, \lambda, 0, \ldots, 0)$ and $r=(-1,0, \lambda, 0,0, \ldots, 0)$. By construction, these points lie on the affine plane $\mathcal{H}=\left\{x_{3}=\lambda, x_{4}=0, \ldots, x_{d}=0\right\}$ as claimed.

Let $\mathrm{t}=(+1, \ldots,+1)$ and $\mathrm{t}^{\prime}=(-1,-1,+1, \ldots,+1)$. By definition of $T O C(\theta)$ we have $\theta_{\mathrm{t}}^{p}=\theta_{\mathrm{t}}^{q}=\theta_{\mathrm{t}}^{r}=\theta$. We use Theorem 5 to determine the total order used at slope $\mathrm{t}^{\prime}$ for the three points: $\theta_{\mathrm{t}^{\prime}}^{p}\left[\mathrm{t}^{\prime} \cdot p, \infty\right)=\theta_{\mathrm{t}}^{p}[\mathrm{t} \cdot p, \infty)-\mathrm{t} \cdot p+\mathrm{t}^{\prime} \cdot p=\theta[\mathrm{t} \cdot p, \infty)-\mathrm{t} \cdot p+\mathrm{t}^{\prime} \cdot p=\theta[\lambda, \infty)$. Similarly, at point $q$ we have $\theta_{\mathrm{t}^{\prime}}^{q}[\lambda+1, \infty)=\theta[\lambda-1, \infty)+2=(\theta+2)[\lambda+1, \infty)$ and at point $r$ we have $\theta_{\mathrm{t}^{\prime}}^{r}[\lambda+1, \infty)=(\theta+2)[\lambda+1, \infty)$ (The six total orders and their relevant orthants are depicted in Figure 5, right).

For any $n>0$ consider the bounded interval $[\lambda, \lambda+n-1]$. We apply Lemma 17 in the third quadrant to obtain relationships between $\theta_{\mathrm{t}^{\prime}}^{p}, \theta_{\mathrm{t}^{\prime}}^{q}$ and $\theta_{\mathrm{t}^{\prime}}^{r}$. Let $X_{1}^{p q}(n), X_{2}^{p q}(n)$, and $X_{3}^{p q}(n)$ be the partition in the three sets obtained when applying the subtree property to $p$ and $q$ (similarly, we define the sets $X_{i}^{p r}$ ). Since we are applying it to the third quadrant and in particular the axis-order is $x_{2}, x_{1}$, we must swap the definitions of $s_{1}$ and $s_{2}$ (i.e., $s_{1}$ will be equal to the difference in the $x_{2}$ coordinate of $p$ and $q$ ).

For the pair $p, q$ we have $s_{1}=1, s_{2}=0$. Thus the left interval consists of the singleton $[\lambda, \lambda]$, the right interval is $[\lambda+1, \lambda+n-1], X_{3}^{p q}(n)=\emptyset$ and we are splitting the numbers of the right interval into sets $X_{1}^{p q}(n)$ and $X_{2}^{p q}(n)$ depending on whether or not they are larger than $\lambda$. That is,

$$
\begin{aligned}
& X_{1}^{p q}(n)=[\lambda+1, \lambda+n-1] \cap\left\{i \in \mathbb{Z}: i \prec \theta_{\mathrm{t}^{\prime}} \lambda\right\}, \\
& X_{2}^{p q}(n)=[\lambda+1, \lambda+n-1] \cap\left\{i \in \mathbb{Z}: \lambda \prec_{\theta_{\mathrm{t}^{\prime}}^{p}} i\right\} .
\end{aligned}
$$




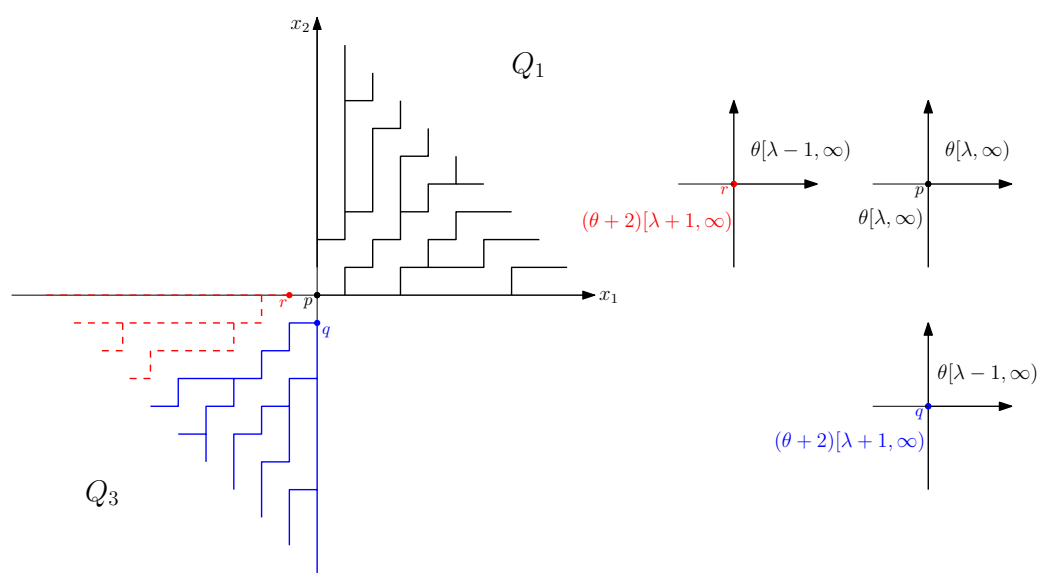

Figure 5 An example of the CDR at $p$ is shown on the left hand side and the relationships between the total orders for the different quadrants at $p, q$ and $r$ on the right hand side. The subtrees at $q$ and at $r$ in $Q_{3}$ are represented by solid blue and dashed red segments respectively. In the example $\theta[0,8]=\{2 \prec 8 \prec 4 \prec 0 \prec 6 \prec 9 \prec 1 \prec 5 \prec 3 \prec 7\}$.

Applying the subtree property to the pair $p, r$ gives a similar partition. In this case, the three sets become $X_{1}^{p r}(n)=\emptyset, X_{2}^{p r}(n)=[\lambda+1, \lambda+n-1] \cap\left\{i \in \mathbb{Z}: i \prec \theta_{t^{\prime}}^{p} \lambda\right\}=X_{1}^{p q}(n)$, and $X_{3}^{p r}(n)=[\lambda+1, \lambda+n-1] \cap\left\{i \in \mathbb{Z}: \lambda \prec_{\theta_{\mathrm{t}^{\prime}}^{p}} i\right\}=X_{2}^{p q}(n)$.

The sets $X_{i}^{p q}$ imply some constraints on $\theta_{\mathrm{t}^{\prime}}^{q}$ (similarly, $X_{i}^{p r}$ gives constraints on $\theta_{\mathrm{t}^{\prime}}^{r}$ ). Recall that we previously observed that $\theta_{\mathrm{t}^{\prime}}^{q}[\lambda+1, \infty)=\theta_{\mathrm{t}^{\prime}}^{p}[\lambda+1, \infty)=(\theta+2)[\lambda+1, \infty)$, which in particular implies that all constraints of the subtree property apply to $\theta+2$.

$X_{2}^{p q}(n)$ says that all relationships in $\theta_{\mathbf{t}^{\prime}}^{p}[\lambda+1, \lambda+n-1]$ are preserved for numbers that are larger than $\lambda$ in $\theta_{\mathrm{t}^{\prime}}^{p}$. Similarly, $X_{2}^{p r}(n)$ says that relationships for numbers smaller than $\lambda$ must also be preserved. Thus, we conclude that all relationships (both larger and smaller than $\lambda)$ must be preserved. Hence, we conclude that $\theta_{\mathrm{t}^{\prime}}^{p}[\lambda+1, \lambda+n-1] \subset(\theta+2)[\lambda+1, \infty)$. This reasoning applies for any values of $\lambda \in \mathbb{Z}$, and $n>0$. In particular, when $\lambda \rightarrow-\infty$ and $n \rightarrow \infty$ we get $\theta=\theta+2$ as claimed.

With this result we can now show Theorem 15 .

(Proof of Theorem 15). Let $\mathrm{t}^{\prime}=(+1, \ldots,+1)$ and note that, by definition, we have $\theta_{\mathrm{t}^{\prime}}^{p}=\theta$. We apply Theorem 5 and obtain $\theta_{\mathrm{t}}^{p}[\mathrm{t} \cdot p, \infty)=\theta_{\mathrm{t}^{\prime}}^{p}\left[\mathrm{t}^{\prime} \cdot p, \infty\right)-\mathrm{t}^{\prime} \cdot p+\mathrm{t} \cdot p=\theta\left[\mathrm{t}^{\prime} \cdot p, \infty\right)-\mathrm{t}^{\prime} \cdot p+\mathrm{t} \cdot p$. The term $-\mathrm{t}^{\prime} \cdot p+\mathrm{t} \cdot p$ must be an even number (since each coordinate of vector $\mathrm{t}-\mathrm{t}^{\prime}$ is either a zero or a two). Thus, we can apply $\theta=\theta+2$ repeatedly until we get $\theta\left[\mathrm{t}^{\prime} \cdot p, \infty\right)-\mathrm{t}^{\prime} \cdot p+\mathrm{t} \cdot p=\theta[\mathrm{t} \cdot p, \infty)$ as claimed.

Specifically, we give two necessary conditions that together are also sufficient. The two conditions are derived from the axioms S1-S5. The first necessary condition is $\theta=\theta+2$, which is already proved in Lemma 18.

The other necessary condition derives from the symmetry axiom (S2) of CDSs.

- Lemma 19 (Necessary condition 2 for CDSs). Any total order such that TOC $(\theta)$ forms a $C D S$ satisfies that $\theta=-(\theta+1)^{-1}$.

Proof (Sketch). This proof follows the same spirit as Theorem 5, but using the symmetry axiom instead. For any two numbers $a, b$ such that such that $a \prec_{\theta} b$ we choose two points $p, q \in \mathbb{Z}^{d}$ and look at $R(p, q)$. In particular, we look at two specific intermediate points $r$ 
and $s$. The key property of these two points is that the behavior of $R(p, q)$ around those points is determined by the positions of $a$ and $b$ in $\theta$. Then, we look at the symmetric path $R(q, p)$ and show that the behavior around the same intermediate points now depends on the positions of $-b-1$ and $-a-1$. In order to satisfy the symmetry axiom, the return path $R(q, p)$ has to be the same and thus we must have $-b-1 \prec_{\theta}-a-1$.

This completes one side of the implication of Theorem 6 . In order to complete the proof we show that the two requirements for $\theta$ are also sufficient.

- Lemma 20 (Sufficient condition for CDSs). Let $\theta$ be a total order that satisfies $\theta+2=\theta$ and $\theta=-(\theta+1)^{-1}$. Then, TOC $(\theta)$ forms a CDS.

\section{Characterization of necessary and sufficient conditions}

Let $\mathcal{F}$ be the collection of total orders of $\mathbb{Z}$ that satisfy the necessary and sufficient conditions of Theorem 6. In order to bound the Hausdorff distance of the CDS associated to these constructions, we must give properties of total orders in $\mathcal{F}$.

- Observation 21. All odd numbers appear monotonically in any total order $\theta$ that satisfies $\theta=\theta+2$. The same holds for even numbers.

The above result follows from repeatedly applying the fact that $a \prec_{\theta} b \Leftrightarrow a+2 \prec_{\theta} b+2$. The second necessary condition also gives a strong relationship between odd and even numbers.

Observation 22. Let $\theta$ be a total order such that $\theta=-(\theta+1)^{-1}$. Then, it holds that $0 \prec_{\theta} 2 \Leftrightarrow-3 \prec_{\theta}-1$.

By combining the previous two observations we get that either both odd and even numbers increase monotonically for any $\theta \in \mathcal{F}$ or both decrease monotonically. Next we study the relationship between odd and even numbers.

Lemma 23. Let $\theta \in \mathcal{F}$ be a total order in which two numbers of the same parity are consecutive in $\theta$. Then, it holds that $1 \prec_{\theta} 2 \Leftrightarrow 2 q+1 \prec_{\theta} 2 q^{\prime}$ for all $q, q^{\prime} \in \mathbb{Z}$.

Corollary 24. There are exactly four total orders in $\mathcal{F}$ in which two numbers of the same parity are consecutive.

Proof. Let $\theta \in \mathcal{F}$ be any such total order. By Lemma 23 either all odd numbers appear before all even numbers or vice versa. There are four cases depending on whether $0 \prec_{\theta} 2$ or $2 \prec_{\theta} 0$ and $1 \prec_{\theta} 2$ or $2 \prec_{\theta} 1$. The first inequality determines whether all even numbers appear monotonically increasing or decreasing in $\theta$ (by Observations 21 and 22 this also determines the order of all odd numbers). The second inequality determines whether odd numbers are smaller or larger (with respect to $\prec_{\theta}$ ) than the even ones. Thus, under the assumption that two numbers of the same parity are consecutive in $\theta$, only the following four orders exist:

$$
\begin{aligned}
\tau_{o^{+} e^{+}} & \{\ldots \prec 1 \prec 3 \prec 5 \prec \ldots \prec 0 \prec 2 \prec 4 \prec \ldots\}, \\
\tau_{o^{-} e^{-}} & \{\ldots \prec 5 \prec 3 \prec 1 \prec \ldots \prec 4 \prec 2 \prec 0 \prec \ldots\}, \\
\tau_{e^{+} o^{+}}=\left(\tau_{o^{-} e^{-}}\right)^{-1}= & \{\ldots \prec 0 \prec 2 \prec 4 \prec \ldots \prec 1 \prec 3 \prec 5 \prec \ldots\}, \\
\tau_{e^{-} o^{-}}=\left(\tau_{o^{+} e^{+}}\right)^{-1}= & \{\ldots \prec 4 \prec 2 \prec 0 \prec \ldots \prec 5 \prec 3 \prec 1 \prec \ldots\} .
\end{aligned}
$$


It remains to consider the case in which $\theta \in \mathcal{F}$ is a total order in which no two numbers of the same parity appear consecutively. That is, we have an odd number followed by an even number, followed by an odd number, and so on. For any $q \in \mathbb{Z}$, let $\alpha_{q}$ be the unique total order satisfying $0 \prec_{\alpha_{q}} 2 q+1 \prec_{\alpha_{q}} 2 \prec_{\alpha_{q}} 2 q+3$ and $\alpha_{q}=\alpha_{q}+2$.

D Theorem 25. $\mathcal{F}=\left\{\tau_{o^{+} e^{+}}, \tau_{o^{-} e^{-}}, \tau_{e^{+} o^{+}}, \tau_{e^{-} o^{-}}\right\} \cup\left\{\alpha_{q}: q \in \mathbb{Z}\right\} \cup\left\{\left(\alpha_{q}\right)^{-1}: q \in \mathbb{Z}\right\}$

This completely characterizes the set $\mathcal{F}$ of total orders, and allows us to find a lower bound on the Hausdorff distance of the associated CDSs.

- Theorem 26. For any $p=\left(p_{1}, \ldots, p_{d}\right) \in \mathbb{Z}^{d}$, total order $\theta \in \mathcal{F}$ and $n>0$, there exists a point $q \in \mathbb{Z}^{d}$ such that $\|p-q\|_{1}=6 n$ and $H(\overline{p q}, R(p, q)) \geq \frac{2 \sqrt{5} n}{5}$.

Proof (Sketch). Pick a point $q$ sufficiently far from $p$ and look at one every other step in the path $R(p, q)$. The way in which the path behaves will depend on the position of the odd numbers of $\theta$ (or even numbers depending on the parity of the starting point). Since odd and even numbers appear monotonically in $\theta$, the path will do all steps in one direction before moving into a different one. Intuitively speaking, the movements in the odd numbers will form a bounding box and so will the movements in the even numbers (although the path is not necessarily the bounding box CDS).

- Remark. First notice that a linear upper bound in the Hausdorff distance trivially follows from the monotonicity axiom. Although asymptotically speaking our construction has the same Hausdorff distance as the bounding box CDS, it can be seen that our leading constant is roughly twice smaller: for points whose $L_{1}$ distance is at most $n$, the bounding box CDS has an error of $\frac{\sqrt{2} n}{4} \approx 0.3 n$ whereas, say, $\operatorname{TOC}\left(\tau_{o^{+} e^{+}}\right)$has an error of $\frac{\sqrt{5} n}{15} \approx 0.15 n$.

\section{Conclusions}

Increasing the dimension from two to three brings a significant change in the associated constraints for creating CDRs and CDSs. Although we have not been able to create a CDS with $o(n)$ Hausdorff distance, we believe that the results presented in this paper provide the first significant step towards this goal. The next natural step would be to consider constructions that apply different total orders to different points of $\mathbb{Z}^{d}$.

For simplicity of exposition, we have defined the CDS as the union of CDRs at all points. The construction of Christ et al. [3] considers the union of half CDRs instead (CDRs that are defined for only half of the slopes, such as slopes that satisfy $t_{1}=+1$ ). We note that the same result would follow if we use their approach. Indeed, in order to derive the two necessary conditions, we have only looked at two slopes. For simplicity we have used $(+1, \ldots,+1)$ and $(-1,-1,+1, \ldots,+1)$, but the same result follows for any two slopes that differ in two coordinates. Thus, constructing CDSs by gluing half CDRs would result in the same necessary and sufficient constraints.

Similarly, one could consider using some kind of priority between slopes (say, lexicographical) so that if $p$ and $q$ are in more than one orthant, only the definition of $R(p, q)$ in the lexicographically smallest slope is considered. This removes the dependency between orthants (Theorem 5), but has a consistency problem: we can find three points $p, q, q^{\prime} \in \mathbb{Z}^{d}$ such that $R(p, q)$ and $R\left(p, q^{\prime}\right)$ have different slopes, but the intersection of the two segments is not connected (such as in Figure 6). 


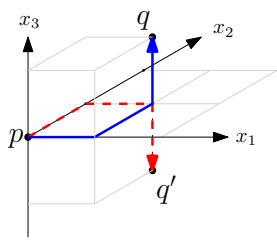

Figure 6 Removing dependency between orthants can create inconsistencies between them.

Acknowledgements. The authors would like to thank Takeshi Tokuyama and Matthew Gibson for their valuable comments during the creation of this paper, as well as the anonymous reviewers whose comments have helped in improving the quality of the paper.

\section{References}

1 Iffat Chowdhury and Matt Gibson. A characterization of consistent digital line segments in $\mathbb{Z}^{2}$. In Proceedings of the 23rd Annual European Symposium on Algorithms, pages 337-348, 2015 .

2 Iffat Chowdhury and Matt Gibson. Constructing consistent digital line segments. In Proceedings of the 12th Latin American Theoretical Informatics Symposium, pages 263-274, 2016.

3 Tobias Christ, Dömötör Pálvölgyi, and Miloš Stojaković. Consistent digital line segments. Discrete \& Computational Geometry, 47(4):691-710, 2012.

4 Jinhee Chun, Matias Korman, Martin Nöllenburg, and Takeshi Tokuyama. Consistent digital rays. Discrete and Computational Geometry, 42(3):359-378, 2009.

5 Michael T. Goodrich, Leonidas J. Guibas, John Hershberger, and Paul J. Tanenbaum. Snap rounding line segments efficiently in two and three dimensions. In Proceedings of the 13th Annual Symposium on Computational Geometry, pages 284-293, 1997.

6 Daniel H. Greene and F. Frances Yao. Finite-resolution computational geometry. In Proceedings of the 27th Annual Symposium on Foundations of Computer Science, pages 143-152, 1986.

$7 \quad$ M. G. Luby. Grid geometries which preserve properties of Euclidean geometry: A study of graphics line drawing algorithms. In NATO Conference on Graphics/CAD, pages 397-432, 1987.

8 Kokichi Sugihara. Robust geometric computation based on topological consistency. In Proceedings of the 9th International Conference on Computational Science, pages 12-26, 2001.

9 Johannes van der Corput. Verteilungsfunktionen I \& II (in german). Proceedings of the Koninklijke Nederlandse Akademie van Wetenschappen, 38:813-820, 1058-1066, 1935. 